\title{
Influência da adição de diatomita nas propriedades de pastas geopoliméricas: volume de filtrado, tempo de espessamento e resistência à compressão
}

\section{(Influence of diatomite addition in the properties of geopolymeric pastes: fluid loss control volume, thickening time and compressive strength)}

\author{
E. A. Santos ${ }^{1}$, M. A. F. Melo ${ }^{1,2}$, D. M. A. Melo ${ }^{1,3}$, J. C. O. Freitas ${ }^{3}$, A. E. Martinelli, \\ V. R. M. Melo ${ }^{3}$, R. L. B. A. Medeiros ${ }^{1}$ \\ IPrograma de Pós-Graduação em Ciência e Engenharia de Materiais, \\ ${ }^{2}$ Departamento de Engenharia Química, \\ ${ }^{3}$ Departamento de Química Universidade Federal do Rio Grande do Norte - UFRN, Natal, RN 59072-970 \\ eylisson@yahoo.com.br,mafm.ufrn@gmail.com,daraujomelo@gmail.com,juliofreitasj@hotmail.com, \\ martinelli.ufrn@gmail.com,rodrigomelo2@hotmail.com,rodolfoluiz.engenharia@hotmail.com
}

\section{Resumo}

Hoje em dia a diversidade de materiais disponíveis para uso em engenharia é muito grande, há um aumento de materiais específicos para cada tipo de aplicação. Os geopolímeros têm sido estudados há décadas; são polímeros inorgânicos que podem proporcionar características de cimentação, que surgem como uma nova classe de materiais de alto desempenho, capazes de substituir total ou parcialmente o cimento Portland em várias aplicações. Neste trabalho foi estudada a influência da adição de diatomita em pastas geopoliméricos baseadas em metacaolinita. A utilização de uma nova matéria-prima abundante na natureza e de baixo valor, como a diatomita, é uma outra alternativa como fonte de sílica para produzir geopolímeros. A diatomita foi usada como um aditivo para melhorar algumas propriedades importantes das pastas de cimento com base em geopolímeros. O efeito da adição de diatomita nas propriedades das pastas geopoliméricas foi avaliado por comparação com as propriedades do geopolímero com base em metacaulim. Para analisar os efeitos de concentrações de diatomita em pastas geopoliméricas, foram realizados testes de filtrado, tempo de espessamento e resistência à compressão. Também são apresentados os resultados de difração de raios X e microscopia electrónica de varredura a fim de reunir informação para uma melhor compreensão da microestrutura do cimento geopolimérico. Os resultados mostraram que a adição de diatomita mudou drasticamente microestrutura e propriedades mecânicas de pastas geopoliméricas. Com o aumento na concentração de diatomita o volume do filtrado diminui, independente da temperatura.

Palavras-chave: pastas geopoliméricas, metacaulim, diatomita.

Abstract

Today, the diversity of available materials for use in engineering is very large, resulting in an increasing of specific materials for each type of application. The geopolymers have been studied for various decades. They are inorganic polymers that can provide cementing characteristics that emerge as a new class of high performance materials, capable of totally or partially replace the Portland cement in various applications. In this work, we studied the influence of the addition of diatomite on geopolymeric pastes based on metakaolin. The use of a new raw material abundant in nature and low value like the diatomite is another alternative as silica source to produce geopolymers. The diatomite is an amorphous silica and in this work it was used as an additive to improve some important properties in cement pastes based in geopolymers. The effect of adding of the diatomite the geopolymeric properties of the slurries was evaluated by comparing them with the properties of the geo-polymer based on metakaolin. To analyze the effects of diatomite concentrations in geopolymeric pastes, filtrate tests were performed, thickening time and compressive strength. Also presents the results of X-ray diffraction (XRD) and scanning electron microscopy (SEM) in order to gather information for a better understanding of the geopolymers cement microstructure. The results showed that the addition of diatomite changed drastically the microstructure and mechanical properties of geopolymer pastes. With the increasing in the concentration of diatomite, the filtrate volume decreases, independent on the temperature.

Keywords: geopolymeric pastes, metakaolin, diatomite.

\section{INTRODUÇ̃̃O}

Diversas são as pesquisas na área de tecnologia de novos materiais cimentantes, visando conferir maior durabilidade através da melhoria das propriedades químicas, físicas ou mecânicas de pastas cimentantes. Isso pode ser feito por meio de emprego de aditivos que apresentam comportamento diferenciado em relação ao tradicional cimento portland, 
material aglomerante mais empregado no mundo. Uma alternativa ao cimento Portland, amplamente estudada nas últimas décadas, consiste no cimento geopolímerico, que é um cimento polimérico inorgânico, também conhecido como polissialato. Esse composto apresenta características particulares que revelam o seu grande potencial de aplicação como aglomerante em substituição total ou parcial ao cimento portland convencional [1]. Os geopolímeros são novos materiais inorgânicos que têm substituído pastas cimentícias, plásticos e determinados produtos minerais [2]. O termo geopolímero foi criado por Davidovits em 1979, para designar uma classe de materiais obtidos por ativação alcalina de precursores à base de silicatos e aluminatos [3]. De acordo com vários pesquisadores, os materiais obtidos por ativação alcalina são, em geral, mais resistentes do ponto de vista mecânico e apresentam maior durabilidade [4]. Quanto ao aspecto de poluição ambiental relacionada à sua fabricação, é considerada ecologicamente mais correta, quando comparada ao cimento Portland. Considerado de alto desempenho, tem encontrado aplicações estruturais e refratárias nas mais diversas áreas, desde as indústrias de construção civil, cerâmica, petroquímica, nuclear, automobilística e naval [5].

As diferentes formas de síntese dos geopolímeros são baseadas nas composições molares entre os compostos reagentes, que influenciam diretamente nas propriedades do produto final obtido. As principais matérias-primas utilizadas são o metacaulim, como fonte de silício e alumínio e a escória granulada de alto-forno, como fonte de cálcio, além de uma fonte de álcalis $(\mathrm{KOH})$ [6]. A fim de se aliar o baixo custo com a questão da preservação ambiental, muitos estudos vêm sendo desenvolvidos para incorporar diversos produtos em pastas de cimento, como por exemplo: resíduos industriais e argilominerais (vermiculita, bentonita e diatomita), reduzindo assim o custo final do produto, além da melhoria das propriedades da pasta. Neste contexto, o desenvolvimento de trabalhos que contemplem pastas geopoliméricas com adição de materiais alternativos torna-se muito importante, dentro de uma visão que trata estes materiais como matéria-prima importante para aplicações nobres, com maior valor agregado. Dessa forma, a utilização de diatomita neste trabalho como aditivo foi incentivada por sua abundância e baixo custo, obtendose pastas geopoliméricas com propriedades adequadas à cimentação de poços de petróleo, se tornando outra alternativa a aplicação de cimento Portland. A utilização de diatomita como aditivo em geopolímeros é uma ideia que surge para viabilizar economicamente a utilização desse material em aplicações de engenharia, buscando assim, mais uma alternativa a pastas de cimento portland, além do desenvolvimento de novos materiais, com boas propriedades tecnológicas.

Neste trabalho foram preparadas pastas geopoliméricas utilizando diatomita como aditivo, estudando-se a influência desse material nas reações de geopolimerização e seu efeito nas propriedades das pastas, comparando-as com as propriedades do geopolímero à base de metacaulim.

\section{EXPERIMENTAL}

As pastas geopoliméricas foram formuladas a partir de estudos preliminares, que serviram de referência para a formulação dos geopolímeros. Neste trabalho utilizou-se a razão $\mathrm{SiO}_{2} / \mathrm{Al}_{2} \mathrm{O}_{3}=3,8$, por ser a que produz cimentos geopoliméricos de maior resistência à compressão $[7,8]$. Procurou-se trabalhar com a razão de $\mathrm{K}_{2} \mathrm{O} / \mathrm{Al}_{2} \mathrm{O}_{3}=1$ de modo a promover a estabilidade da rede geopolimérica, pois razões inferiores a 1 podem resultar em polimerização incompleta da rede [4]. Utilizou-se a razão $\mathrm{H}_{2} \mathrm{O} / \mathrm{K}_{2} \mathrm{O}=12$ de forma a obter uma boa homogeneidade, sem excesso de fluidez [8].

Como precursor geopolimérico foi utilizado um metacaulim comercial previamente calcinado (Metacaulim do Brasil Ind. Com.), sendo esta a principal fonte de sílica $\left(\mathrm{SiO}_{2}\right)$ e de alumina $\left(\mathrm{Al}_{2} \mathrm{O}_{3}\right)$. Como fonte adicional de sílica foi utilizado o silicato de potássio (Diatom Miner. Ltda.). Além de participar da ativação alcalina do precursor geopolimérico, o silicato de potássio contribuiu com um teor significativo de sílica extra para a reação. Além de ativador alcalino, hidróxido de potássio (Vetec) foi usado para assegurar o $\mathrm{pH}$ básico necessário à geopolimerização. Como fonte alternativa de sílica utilizou-se a diatomita (Dianorte Ltda.).

A caracterização inicial do metacaulim, da diatomita e do silicato de potássio foi fundamental para a determinação da composição química desses materiais e posterior cálculo das pastas geopoliméricas. No estudo de geopolímeros, a análise química é de extrema importância uma vez que obtém-se os percentuais de $\mathrm{SiO}_{2}, \mathrm{Al}_{2} \mathrm{O}_{3}$ e $\mathrm{M}_{2} \mathrm{O}$ do precursor e do aditivo, $\mathrm{M}$ representa o íon $\mathrm{K}^{+}$ou $\mathrm{Na}^{+}$. Diante desses dados, acrescidos das informações sobre as concentrações nos ativadores, estabelece-se a quantidade de material a ser trabalhado na formulação dos geopolímeros, ou seja, as razões molares $\mathrm{SiO}_{2} / \mathrm{Al}_{2} \mathrm{O}_{3}$ definidas em cada formulação, assim como as razões $\mathrm{M}_{2} \mathrm{O} / \mathrm{Al}_{2} \mathrm{O}_{3}$. A composição química dos materiais foi determinada por meio de análise química semiquantitativa em um espectrômetro de fluorescência de raios X Shimadzu EDX-720, sendo as amostras sólidas (metacaulim e diatomita) analisadas sob vácuo. A composição química do silicato de potássio foi realizada por titulação, apresentando cerca de $62 \%$ de umidade.

A distribuição granulométrica das amostras de metacaulim e da diatomita foi obtida em um granulômetro a laser Cilas 920. Esta análise se faz necessária, pois se estima o grau de participação dos componentes em função do tamanho das partículas.

Para encontrar a massa correspondente de metacaulim e de diatomita necessárias para se colocar na mistura, foi necessário determinar a massa específica de ambos constituintes. As massas específicas do metacaulim e da diatomita foram obtidas pelo método de "Le Chatelier" [9]. As medidas de massa específica foram feitas utilizandose etanol absoluto como solvente. A medida da massa específica do silicato de potássio foi feita em uma balança de lama atmosférica Baroid [10]. Foram realizadas três 
determinações por amostra, obtendo-se o resultado pela média.

Para avaliar a influência da adição de diatomita em pastas geopoliméricas, os cálculos foram feitos mantendose constante a razão molar de $\mathrm{SiO}_{2} / \mathrm{Al}_{2} \mathrm{O}_{3}=3,8$, utilizada na pasta geopolimérica à base de metacaulim. Assim, para atender a tais condições, a diatomita teve sua composição química definida para que então pudesse ser complementado com os teores adequados de metacaulim e hidróxido de potássio. As proporções entre as matérias primas foram determinadas para que todas as composições atingissem as razões molares $\mathrm{SiO}_{2} / \mathrm{Al}_{2} \mathrm{O}_{3}=3,8$ e $\mathrm{K}_{2} \mathrm{O} / \mathrm{Al}_{2} \mathrm{O}_{3}=1$. Inicialmente, fez-se a incorporação da maior quantidade de diatomita possível na pasta geopolimérica à base de metacaulim, até uma quantidade que não comprometesse a manipulação das pastas no misturador, principalmente, a reologia da pasta fluida e as propriedades do geopolímero, analisando-se também a miscibilidade dos componentes. Neste estudo formulou-se quatro pastas: uma referência (sem adição de diatomita) e outras três com adições de $0,5 \%, 1,0 \%$ e $1,5 \%$ de diatomita. As pastas geopoliméricas foram preparadas dissolvendo-se, inicialmente, o hidróxido de potássio no silicato de potássio, em um becker com um bastão de vidro, formando a solução alcalina. Em seguida, a mistura da pasta foi feita em um misturador Chandler 30-60 contendo a solução alcalina, adicionando-se o metacaulim e a diatomita. O tempo de adição foi controlado pelo temporizador do misturador, sendo em seguida desligado automaticamente [11].

Análises de difração de raios $\mathrm{X}$ do metacaulim e da diatomita foram feitas com o objetivo de comprovar o caráter amorfo destes materiais, pois para que a reação de geopolimerização ocorra é necessário que o metacaulim seja amorfo. Difratogramas de raios $\mathrm{X}$ de amostras das pastas geopoliméricas também foram obtidos a fim de investigar a presença de fases não reativas e possível aumento da amorfização das amostras nos geopolímeros obtidos com a adição de diatomita. Foram escolhidas pastas com concentrações de $1,0 \%$ e $1,5 \%$ de diatomita, comparandoas com a pasta à base de metacaulim. Essas amostras foram escolhidas por apresentarem maior concentração de diatomita, o que levou a maiores mudanças nas propriedades das pastas.

$\mathrm{O}$ estudo da microestrutura por microscopia eletrônica de varredura foi feito em um microscópio Philips XL-30ESEM, operando a $20 \mathrm{kV}$ e utilizando detector de elétrons secundários em módulo a vácuo. As amostras de cimento geopolimérico foram preparadas a partir dos corpos de prova ensaiados por resistência à compressão e consistiu na deposição de fragmentos do centro dos corpos de prova. Além da caracterização morfológica das pastas geopoliméricas, também foram caracterizados o precursor geopolimérico (metacaulim) e o aditivo (diatomita).

\section{RESULTADOS E DISCUSSÃO}

Os resultados das análises químicas e físicas dos materiais utilizados nas pastas geopoliméricas são apresentadas nas Tabelas I e II. Na Tabela I é apresentada a composição química e as massas específicas dos materiais. $\mathrm{O}$ metacaulim comercial é o precursor e fornecedor de aluminosilicatos $\left(\mathrm{Al}_{2} \mathrm{O}_{3}\right.$ e $\left.\mathrm{SiO}_{2}\right)$, necessários para a reação de geopolimerização. Constatou-se que possui $92,94 \%$ destes dois óxidos, apresentando elevados teores de $\mathrm{SiO}_{2}$ e $\mathrm{Al}_{2} \mathrm{O}_{3}$, principal requisito para um material ser considerado bom precursor na geopolimerização. Já a diatomita, utilizada como aditivo, apresenta um teor de aluminosilicatos maior que a do metacaulim, com $95,63 \%$, tendo uma alta concentração de sílica $(84,61 \%)$, porém, baixa concentração de alumina $(11,02 \%)$, se comparada ao metacaulim. No que diz respeito à razão molar $\mathrm{SiO}_{2} / \mathrm{Al}_{2} \mathrm{O}_{3}$, observa-se que os teores de sílica e alumina do metacaulim satisfazem às condições estabelecidas como mínimas para precursores com potencial de ativação geopolimérica, evidenciados pela relação molar $\mathrm{SiO}_{2} / \mathrm{Al}_{2} \mathrm{O}_{3}$ maior do que 1 . O maior valor dessa razão, ou menor quantidade de alumina, apresentada pela diatomita em relação à caulinita (Tabela I), indica que o componente

Tabela I - Composição química e física dos materiais utilizados na preparação das pastas.

[Table I - Chemical and physical composition of the materials used in the preparation of pastes.]

\begin{tabular}{cccc}
\hline Componentes & $\begin{array}{c}\text { Metacaulim } \\
(\%)\end{array}$ & $\begin{array}{c}\text { Diatomita } \\
(\%)\end{array}$ & $\begin{array}{c}\text { Silicato de } \\
\text { potássio } \\
(\%)\end{array}$ \\
\hline $\mathrm{SiO}_{2}$ & 48,74 & 84,61 & 26,18 \\
$\mathrm{Al}_{2} \mathrm{O}_{3}$ & 44,20 & 11,02 & - \\
$\mathrm{Fe}_{2} \mathrm{O}_{3}$ & 2,28 & 1,41 & - \\
$\mathrm{K}_{2} \mathrm{O}$ & 1,94 & 0,10 & 12,17 \\
$\mathrm{SO}_{3}$ & 1,38 & 2,49 & - \\
$\mathrm{TiO}_{2}$ & 1,37 & - & - \\
$\mathrm{CaO}$ & - & 0,24 & - \\
$\mathrm{M} . \mathrm{E} .\left(\mathrm{g} / \mathrm{cm}^{3}\right)$ & 2,52 & 2,20 & 1,37 \\
$\mathrm{SiO} / \mathrm{Al}_{2} \mathrm{O}_{3}$ & 1,10 & 7,68 & - \\
\hline
\end{tabular}

Tabela II - Valores obtidos de análise granulométrica das amostras de metacaulim e diatomita.

[Table II - Particle size analysis of samples of diatomite and metakaolin.]

\begin{tabular}{cccl}
\hline Amostras & $10 \%$ & $50 \%$ & \multicolumn{1}{c}{$90 \%$} \\
\hline \multirow{2}{*}{ Metacaulim } & $\begin{array}{l}\text { Diâmetro } \\
<1 \mu \mathrm{m}\end{array}$ & $\begin{array}{l}\text { Diâmetro } \\
<7 \mu \mathrm{m}\end{array}$ & $\begin{array}{l}\text { Diâmetro } \\
<17 \mu \mathrm{m}\end{array}$ \\
\multirow{2}{*}{ Diatomita } & $\begin{array}{l}\text { Diâmetro } \\
<3 \mu \mathrm{m}\end{array}$ & $\begin{array}{l}\text { Diâmetro } \\
<12 \mu \mathrm{m}\end{array}$ & $\begin{array}{l}\text { Diâmetro } \\
<14 \mu \mathrm{m}\end{array}$ \\
\hline
\end{tabular}


mineral principal não é caulinita $\left(\mathrm{Al}_{2} \mathrm{Si}_{2} \mathrm{O}_{5}(\mathrm{OH})_{4}\right)$. Dessa forma, o geopolímero com diatomita pode apresentar um comportamento diferente perante a ativação alcalina, como diferentes estruturas e resistência mecânica, em relação ao geopolímero à base de metacaulim.

Na Tabela II é mostrada a distribuição granulométrica das amostras de metacaulim e da diatomita. A distribuição dos tamanhos das partículas de metacaulim mostram que $10 \%$ das partículas possuem diâmetros inferiores a $1 \mu \mathrm{m}$, $50 \%$ das partículas possuem diâmetros inferiores a $7 \mu \mathrm{m}$, $90 \%$ das partículas possuem diâmetros inferiores a $17 \mu \mathrm{m}$, e diâmetro médio das partículas de $8 \mu \mathrm{m}$. Já a distribuição dos tamanhos das partículas de diatomita mostram que $10 \%$ das partículas possuem diâmetros inferiores a $3 \mu \mathrm{m}, 50 \%$ das partículas possuem diâmetros inferiores a $12 \mu \mathrm{m}, 90 \%$ das partículas possuem diâmetros inferiores a $29 \mu \mathrm{m}$, e diâmetro médio das partículas de $14 \mu \mathrm{m}$. Informações estas que mostram que os materiais são bastante finos, garantindo uma boa superfície de contato para o meio reacional, participando assim ativamente da reação.

Os resultados do ensaio de volume de filtrado, em função da temperatura, estão apresentados na Fig. 1. Para a realização deste ensaio as pastas foram preparadas e homogeneizadas [11] a $27{ }^{\circ} \mathrm{C}$ e $52{ }^{\circ} \mathrm{C}$. Observou-se que independente da temperatura do ensaio, ao se adicionar diatomita, o volume de filtrado é reduzido de forma linear. Essa redução de volume de filtrado se deu devido a grande capacidade de absorção de água da diatomita, devido à porosidade que este material apresenta, acarretando assim, maior retensão de água da pasta. Como uma esponja rígida, a diatomita reduz o volume de filtrado por efeito físico e químico, através do aumento das camadas filtrantes. Se compararmos a pasta à base de metacaulim com a de 1,5\% de diatomita, verifica-se que a redução no volume de filtrado chegou a $30 \%$ e $42 \%$, respectivamente em $27{ }^{\circ} \mathrm{C}$ e $52{ }^{\circ} \mathrm{C}$. A redução no volume de filtrado à medida que se adiciona

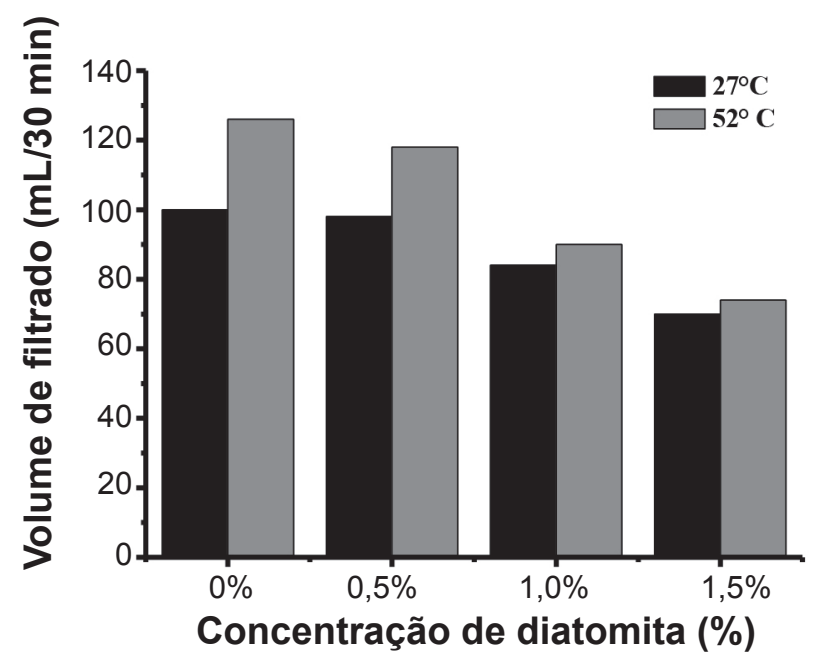

Figura 1: Volume de filtrado em função da concentração de diatomita, nas temperaturas ambiente $\left(27^{\circ} \mathrm{C}\right)$ e aquecida $\left(52{ }^{\circ} \mathrm{C}\right)$. [Figure 1: Fluid loss control as a function of the concentration of diatomite, at room temperature $\left(27^{\circ} \mathrm{C}\right)$ and heated $\left(52^{\circ} \mathrm{C}\right)$.] diatomita reflete em uma melhor formação do reboco e, consequentemente, na redução da desidratação prematura de todas as pastas estudadas, se comparadas ao da pasta à base de metacaulim.

Os resultados dos ensaios de resistência à compressão realizados neste trabalho são mostrados na Fig. 2, e revelam que a adição de diatomita em pastas geopoliméricas resultam em aumentos significativos nos valores de resistência à compressão, em relação a pasta à base de metacaulim, independente do tempo de cura. De modo geral, o avanço do tempo de cura contribuiu positivamente para o aumento significativo da resistência, devido à ocorrência simultânea das reações químicas com as reações de geossíntese ao longo do tempo. $\mathrm{O}$ aumento da concentração de diatomita também leva a um aumento da resistência à compressão. Todas as pastas geopoliméricas com adição de diatomita produziram geopolímeros com resistências mecânicas superiores a dos geopolímeros à base de metacaulim.

Os valores de resistência à compressão mais altos, em relação as pastas à base de metacaulim, aconteceram com $1,5 \%$ de diatomita, para todos os tempos de cura estudados. A adição de 1,5\% de diatomita, curada a 7 dias, aumentou em $67 \%$ a resistência à compressão em relação ao geopolímero à base de metacaulim, chegando a uma resistência de 30,64 $\mathrm{MPa}$, sendo este o maior valor obtido para o geopolímero. Ainda considerando as pastas com 1,5\% de diatomita, observamos que os aumentos da resistência à compressão das pastas curadas a $48 \mathrm{~h}$ e a 7 dias, em relação à pasta curada a $24 \mathrm{~h}$, chegaram a $64 \%$ e $124 \%$, respectivamente. A esse aumento na resistência, podemos associá-lo a área superficial e a inércia química da diatomita. A diatomita é um mineral leve, de baixa massa específica com estrutura porosa, a qual the confere uma elevada área superficial específica. A elevada área superficial da diatomita contribuiu para o aumento nos valores de resistência à compressão, devido a maior reatividade da diatomita com a solução ativadora (silicato de potássio + hidróxido de potássio), pois a mesma possui grande quantidade de sílica reativa. Esse comportamento indica que o aumento da resistência com a adição de diatomita está relacionado com o efeito da solução ativadora alcalina na estrutura da diatomita, que conduz à formação do gel aluminossilicato alcalino com cadeias poliméricas extensas.

O menor valor de resistência à compressão das pastas à base de metacaulim foi $1,29 \mathrm{MPa}$, enquanto que nas pastas com adição de diatomita foi de $3,51 \mathrm{MPa}$ (para $0,5 \%$ diatomita), ambas curadas a $24 \mathrm{~h}$. Esse comportamento das pastas mostra que para essas formulações, neste tempo de cura, há necessidade de um tempo de cura mais longo para realizar a medida de compressão, uma vez que as pastas apresentavam aspecto úmido.

É necessário levar em consideração a ativação térmica das fontes sólidas de $\mathrm{SiO}_{2}$ e $\mathrm{Al}_{2} \mathrm{O}_{3}$ para produzir geopolímeros com aplicações diferentes [12]. A pasta com adição de 1,5\% de diatomita, curada a 7 dias, resultou no geopolímero mais resistente, apresentando uma resistência à compressão de aproximadamente $31 \mathrm{MPa}$, podendo ter aplicação como 


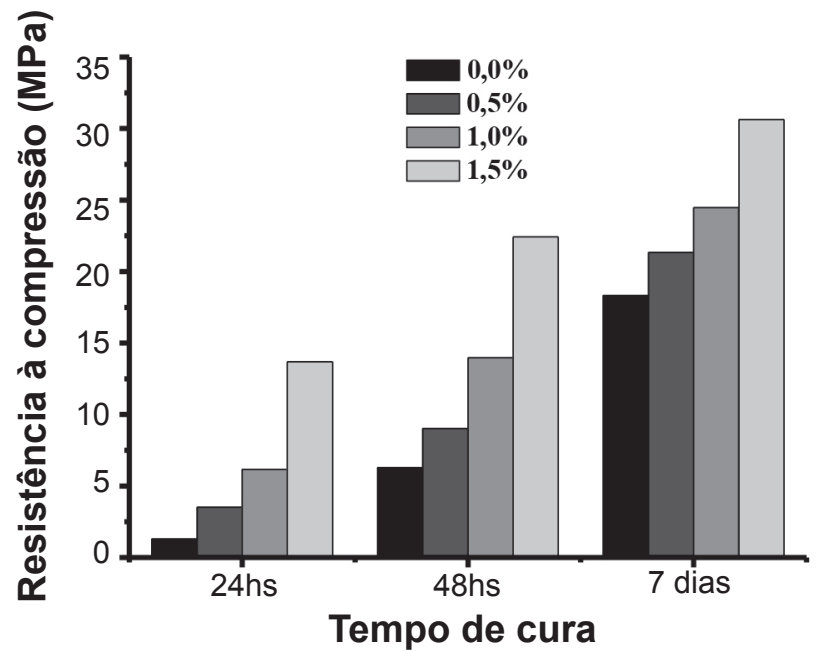

Figura 2: Resistência à compressão em função do tempo de cura das pastas.

[Figure 2: Compressive strength as a function of the curing time of pastes.]

cimento de desempenho mais elevado.

Para a realização do ensaio de tempo de espessamento, escolheu-se as pastas com concentrações de $1,0 \%$ e $1,5 \%$ de diatomita, comparando-as com a pasta à base de metacaulim. Essas amostras foram escolhidas por apresentarem maior concentração de diatomita, o que levou a maiores mudanças nas propriedades das pastas.

Os resultados obtidos dos ensaios de consistometria das pastas (Tabela III) mostram que a adição de diatomita reduz, de forma significativa, o tempo de espessamento das pastas geopoliméricas. Tomando como base o parâmetro do tempo de espessamento das pastas (100 Uc), observase que esse tempo apresenta comportamento semelhante ao de volume de filtrado, onde há uma redução no valor do tempo de espessamento das pastas, a medida que se aumenta a concentração de diatomita, o que leva ao aumento da velocidade na reação que favorece o menor tempo de repouso da pasta e, consequentemente, o menor tempo de espessamento. Esse comportamento está relacionado a vários fatores. Um desses fatores é redução na mobilidade das cadeias geopoliméricas, com a adição de diatomita, provocado pela elevada área superficial dos sólidos, que resultou no aumento dos valores de resistência à compressão. Outro fator que explica este comportamento é a grande

Tabela III - Tempo de espessamento das pastas geopoliméricas.

[Table III - Thickening time of geopolymeric pastes.]

\begin{tabular}{ccc}
\hline $\begin{array}{c}\text { Concentração de } \\
\text { Diatomita }\end{array}$ & $50 \mathrm{Uc}$ & $100 \mathrm{Uc}$ \\
\hline $0,0 \%$ & $5 \mathrm{~h} 27 \mathrm{~min}$ & $8 \mathrm{~h} 10 \mathrm{~min}$ \\
$1,0 \%$ & $2 \mathrm{~h} 15 \mathrm{~min}$ & $2 \mathrm{~h} 34 \mathrm{~min}$ \\
$1,5 \%$ & $1 \mathrm{~h} 30 \mathrm{~min}$ & $1 \mathrm{~h} 40 \mathrm{~min}$ \\
\hline
\end{tabular}

reatividade da diatomita com a solução ativadora (silicato de potássio + hidróxido de potássio), pois a mesma possui grande quantidade de sílica reativa, como já foi mencionado sendo uma das principais causas do aumento da resistência à compressão.

Na Fig. 3 são mostrados os difratogramas de raios $\mathrm{X}$ do metacaulim e da diatomita, utilizados nas pastas geopoliméricas. No caso do metacaulim, a presença da fase amorfa é importantíssima, pois para que a reação de geopolimerização ocorra é necessário que o metacaulim tenha um caráter amorfo. A Fig. 3a apresenta o difratograma de raios $\mathrm{X}$ do metacaulim, onde se pode observar que o mesmo apresenta elevado caráter amorfo, o que favorece a reação de geopolimerização. Porém, ainda foi possível a identificação da fase caulinita $\left(\mathrm{Al}_{2} \mathrm{Si}_{2} \mathrm{O}_{5}(\mathrm{OH})_{4}\right)$, com picos em $2 \theta=12,2964^{\circ} ; 19,7870^{\circ} ; 24,8660^{\circ}$ e $26,5925^{\circ}$, mostrando que a transformação de caulinita em metacaulinita não se deu por completo. A diatomita (Fig. 3b) também apresenta caráter amorfo, com picos de quartzo $\left(\mathrm{SiO}_{2}\right)$, assim como picos de caulinita, além de uma barriga correspondente ao material amorfo presente na amostra, característico da
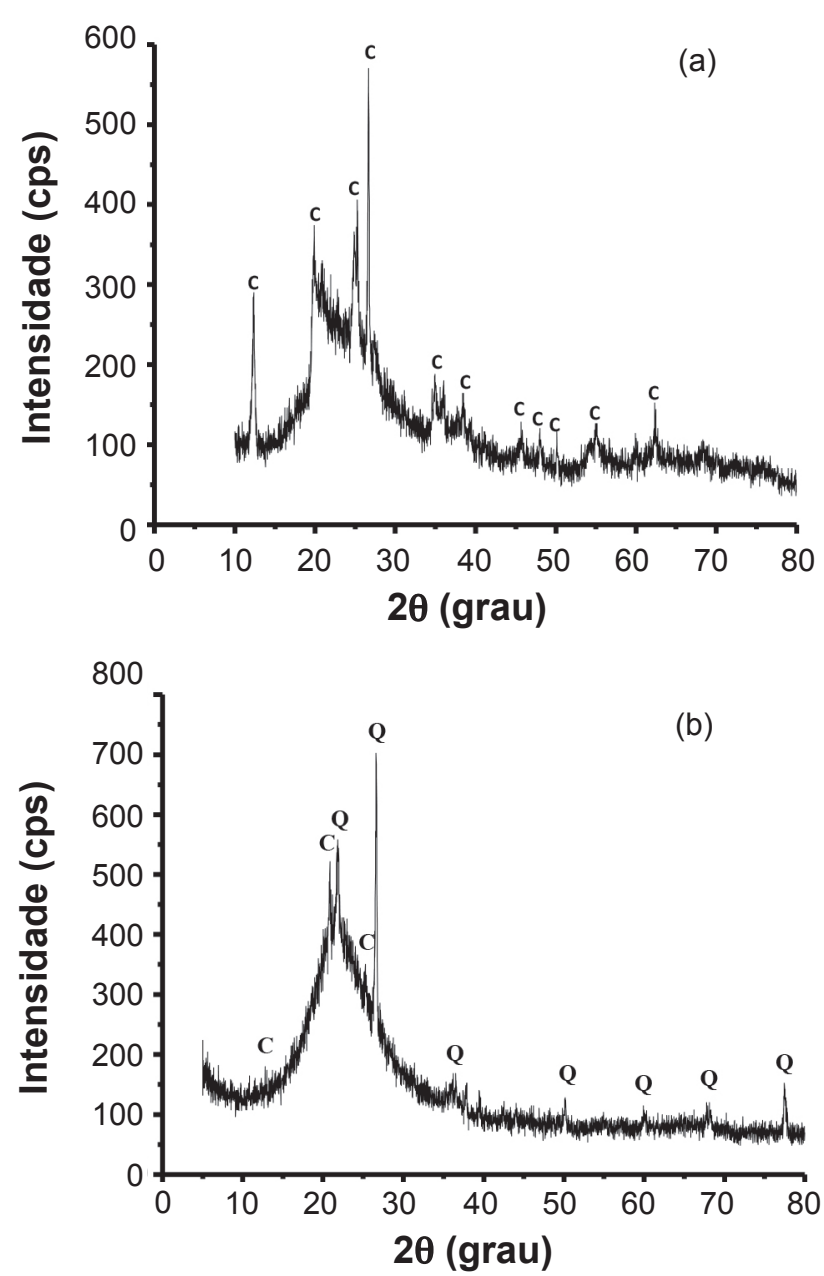

Figura 3: Difratogramas de raios X: (a) metacaulim, (b) diatomita. [Figure 3: X-ray diffraction patterns: (a) metakaolin, (b) diatomite.] 
diatomita que é considerada uma sílica amorfa.

A Fig. 4 apresenta os resultados das análises de difração de raios $\mathrm{X}$ das pastas à base de metacaulim e das pastas com adição de $1,0 \%$ e $1,5 \%$ de diatomita, curadas a $38{ }^{\circ} \mathrm{C}$ após 48 h. Em geral, a adição de diatomita em pastas à base de metacaulim não influenciou na estrutura das pastas, uma vez
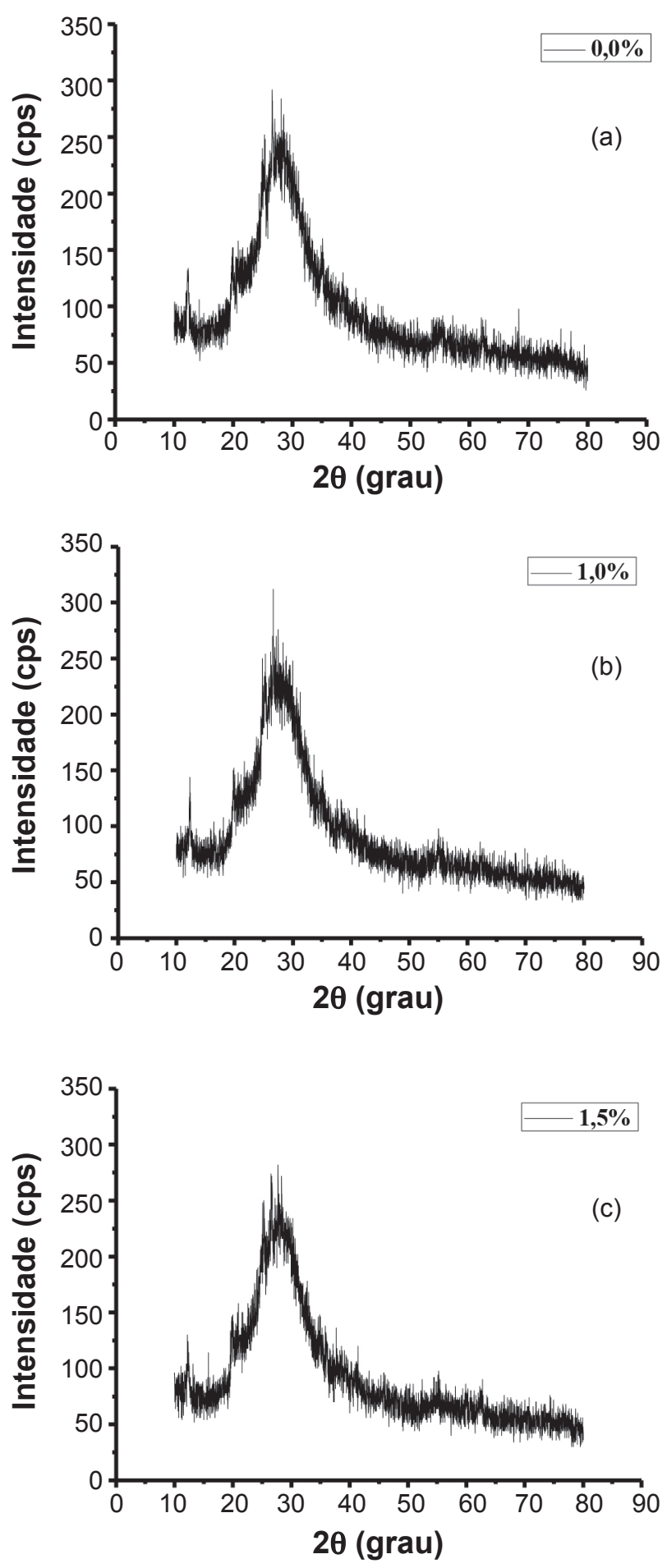

Figura 4: Difratogramas de raios $\mathrm{X}$ das pastas curadas a $38^{\circ} \mathrm{C}$ após $48 \mathrm{~h}$.

[Figure 4: X-ray diffraction patterns of the pastes cured at $38^{\circ} \mathrm{C}$, after $48 \mathrm{~h}$.] que, tanto o metacaulim quanto a diatomita são materiais amorfos. Observou-se uma estrutura predominantemente amorfa para a matriz geopolimérica. Diante disto, escolheuse apenas as pastas curadas a $48 \mathrm{~h}$ para representar esta evidência, uma vez que todas as pastas restantes apresentaram a mesma estrutura amorfa. Em todos os casos, a formação de uma estrutura amorfa é identificada pelo aumento da desordem com $2 \theta$ entre $20^{\circ}$ e $30^{\circ}$.

As micrografias do metacaulim e da diatomita obtidas por MEV são mostradas na Fig. 5, evidenciando a distribuição dos grãos sólidos. O metacaulim (Fig. 5a), apresenta grãos com geometria pouco regular. Já no aspecto morfológico da diatomita (Fig. 5b), observa-se que as frústulas diatomáceas intactas possuem formato cilíndrico e navicular, além de apresentar poros circulares e elípticos, os quais designam a elevada propriedade filtrante deste material.

Para a análise microestrutural das pastas foi escolhida a amostra com a maior concentração de diatomita $(1,5 \%)$, comparando-a com a amostra à base de metacaulim, no tempo de cura de 7 dias, a $38^{\circ} \mathrm{C}$. A amostra com $1,5 \%$ de diatomita foi escolhida por apresentar a maior resistência
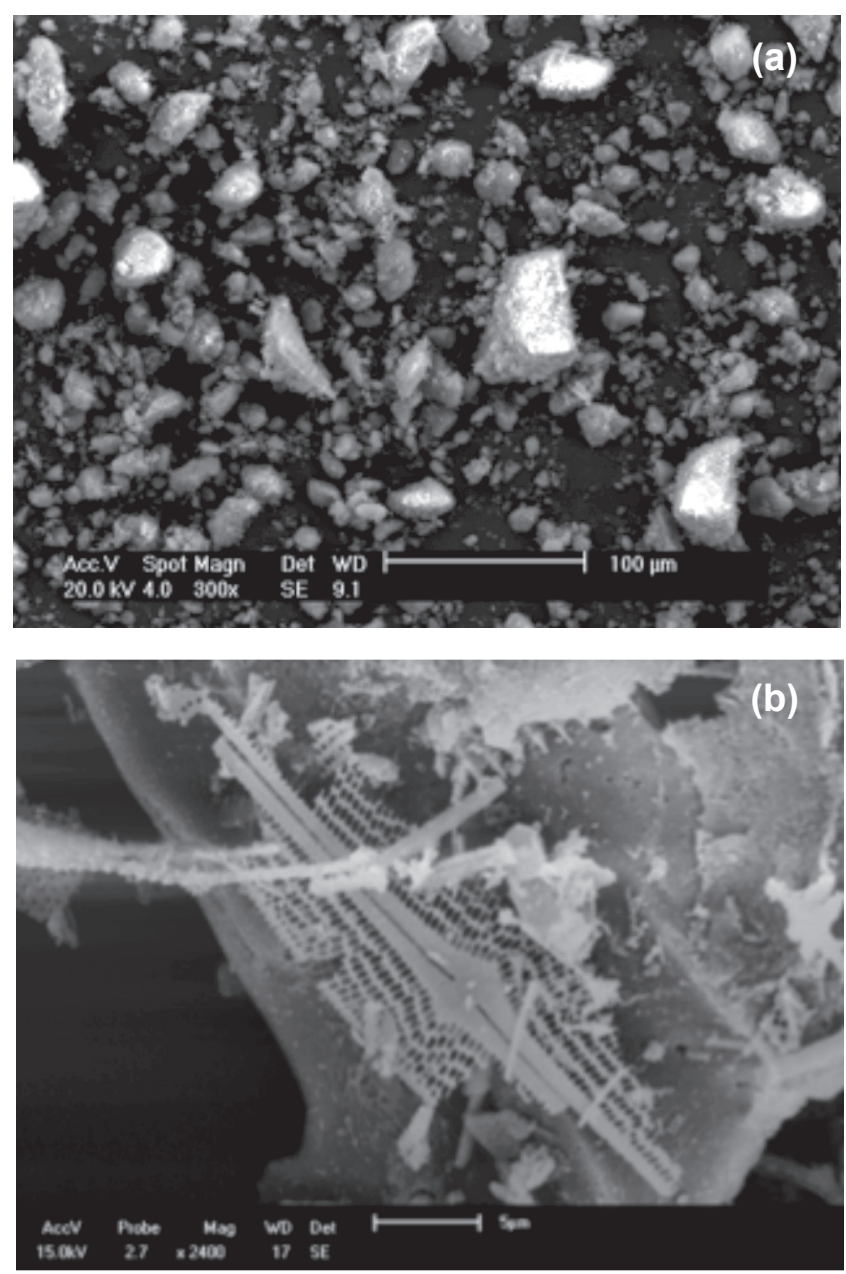

Figura 5: Micrografias obtidas em microscópio eletrônico de varredura: (a) detalhe do metacaulim, (b) detalhe da diatomita.

[Figure 5: SEM micrographs: (a) detail of metakaolin, (b) detail of the diatomite.] 

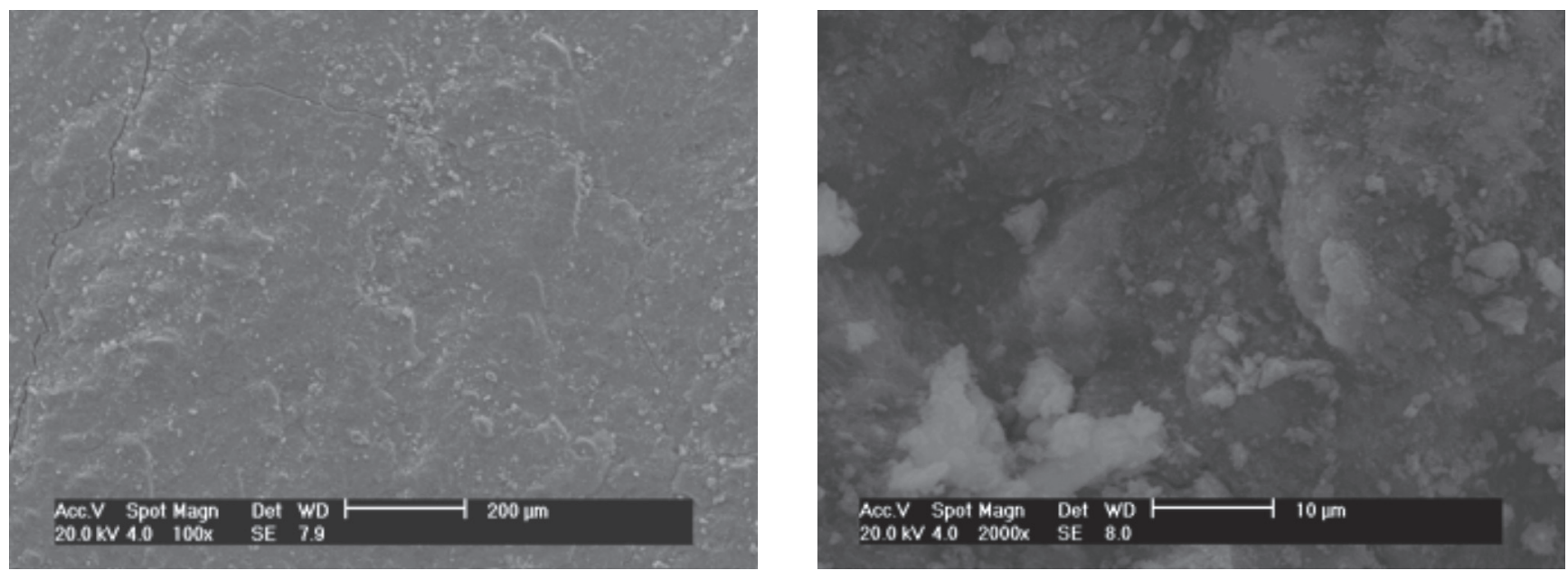

Figura 6: Micrografias obtidas em microscópio eletrônico de varredura das pastas curadas por 7 dias a $38{ }^{\circ} \mathrm{C}$, com $0 \%$ de diatomita. [Figure 6: SEM micrographs of pastes cured for 7 days at $38^{\circ} \mathrm{C}$, with $0 \%$ of diatomite.]
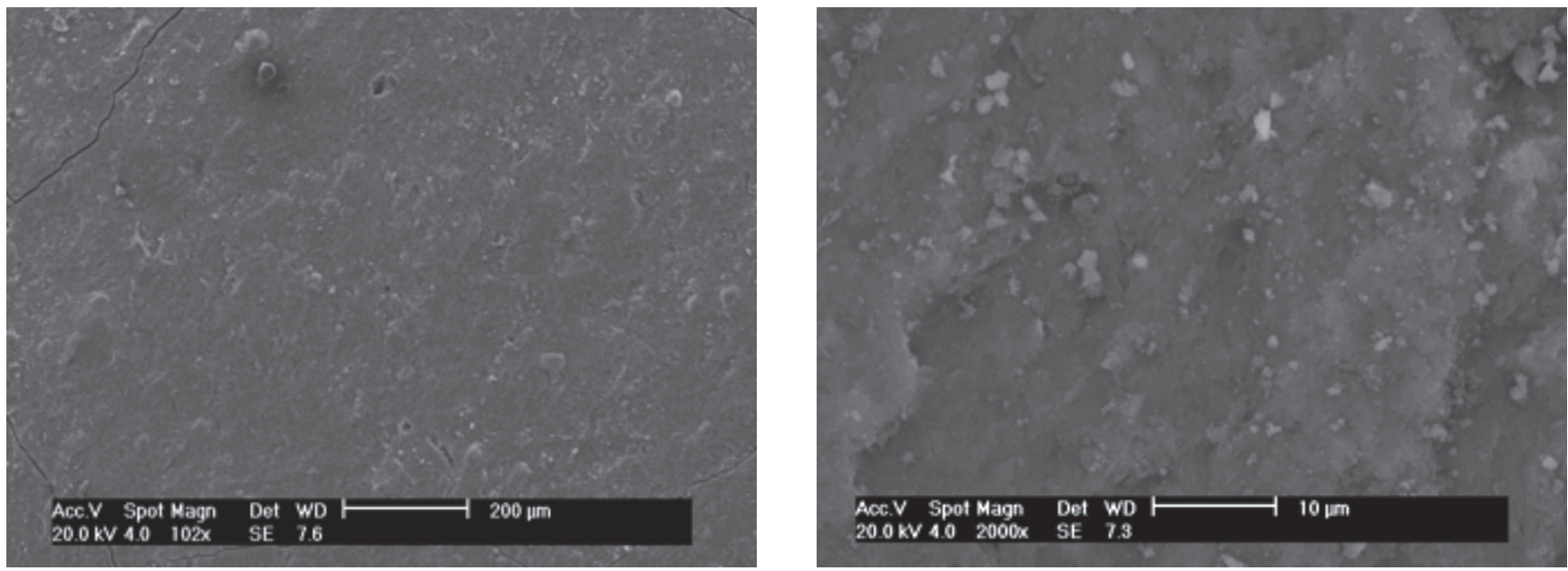

Figura 7: Micrografias obtidas em microscópio eletrônico de varredura das pastas curadas por 7 dias a $38{ }^{\circ} \mathrm{C}$, com $1,5 \%$ de diatomita. [Figure 7: SEM micrographs of pastes cured for 7 days at $38^{\circ} \mathrm{C}$, with $1.5 \%$ of diatomite.]

à compressão. As diferenças na microestrutura das pastas geopoliméricas podem ser observadas nas Figs. 6 e 7, onde são ilustradas as análises das superfícies de fratura das pastas com $0 \%$ de diatomita e $1,5 \%$ de diatomita, respectivamente. As imagens da coluna esquerda apresentam ampliações relativas de 100x e as colunas da direita de 2000x.

Analisando-se e comparando as pastas à base de metacaulim com as pastas com $1,5 \%$ de diatomita, verificouse que ambas apresentaram morfologia semelhante. A presença de trincas encontradas nas amostras pode ser justificada, uma vez que, foram analisados fragmentos retirados da mesma região (após o ensaio de resistência à compressão), ou seja, do centro do corpo de prova. Comparando-se a pasta com adição de diatomita com a pasta à base de metacaulim, observa-se uma pequena diferença na homogeneidade destas. A essa diferença de homogeneidade, podemos associá-la a diferentes reações de superfície no processo de geopolimerização com a presença da diatomita, pois as reações de superfície no processo de geopolimerização dependem de fatores fundamentais como: mineralogia, teor de sílica e alumina, finura ou área superficial reativa e morfologia. A adição de diatomita torna a matriz mais homogênea reduzindo a rugosidade das pastas, além da formação de uma matriz mais densa, compacta e homogênea, contribuindo assim para a elevação da resistência das pastas. A homogeneização e redução na rugosidade das pastas, foi melhor observada nas formulações com a adição de $1,5 \%$ de diatomita, comprovando que, a adição desta contribui para uma melhor estabilidade e fortalecimento da matriz. Ao fazer parte da estrutura geopolimérica, a diatomita serviu para fortalecer a nova estrutura formada.

\section{CONCLUSÕES}

A adição de diatomita em pastas à base de metacaulim levou a uma melhoria significativa das propriedades mecânicas das pastas curadas, aumentando a resistência à compressão em até $67 \%$, devido à área superficial e à inércia 
química da diatomita. Em estudos já realizados verificouse que geopolímeros que apresentaram baixas resistências à compressão estavam relacionadas com baixa razão $\mathrm{SiO}_{2} /$ $\mathrm{Al}_{2} \mathrm{O}_{3}$ e microestrutura de poros grosseiros interconectados, onde o aumento dessa razão acarretou em um aumento da resistência à compressão, e refinamento da estrutura de poros [7]. A razão $\mathrm{SiO}_{2} / \mathrm{Al}_{2} \mathrm{O}_{3}=3,8$ utilizada neste trabalho seguiu esta tendência, confirmado pelos altos valores de resistência à compressão e pela presença de poucos poros na matriz geopolimérica. A diatomita atuou como controlador de filtrado, uma vez que a mesma reduziu a desidratação prematura de todas as pastas, se comparadas a da base de metacaulim, devido a retenção de água da pasta pela diatomita. Observou-se o efeito da redução do tempo de espessamento das pastas com a adição de diatomita, devido a interferência da mesma na reação de geopolimerização das pastas. Essa redução contribuiu para o aumento da resistência à compressão inicial das pastas, devido a grande reatividade da diatomita com a solução ativadora, podendo trazer benefícios como referência à estabilidade do poço. Realizaram-se caracterizações detalhadas nas matérias primas e nas pastas curadas, comprovando a qualidade das matérias-primas utilizadas. Os teores de sílica e alumina do metacaulim satisfazem as condições estabelecidas como mínimas para precursores com potencial de ativação geopolimérica, evidenciado pela relação molar $\mathrm{SiO}_{2} / \mathrm{Al}_{2} \mathrm{O}_{3}$ maior do que 1 . No que diz respeito a difração de raios $X$ das pastas geopoliméricas com adição de diatomita, observouse uma estrutura predominantemente amorfa da matriz geopolimérica. As análises de microscopia eletrônica de varredura mostraram diferença de textura das pastas com a adição de diatomita, em comparação às pastas à base de metacaulim, com formação de geopolímeros com estrutura homogênea, compacta e uniforme, o que contribuiu para a elevação da resistência mecânica e melhor acabamento das amostras.

\section{AGRADECIMENTOS}

Ao LABCIM e LABTAM-UFRN, onde todos os ensaios foram realizados, e à CAPES e ao PPGCEM pela concessão da bolsa de doutorado a E. A. Santos.

\section{REFERÊNCIAS}

[1] F. J. Silva, M. C. Oliveira, M. V. S. Machado, F. P. Duarte, C. Thaumaturgo, "Cimentos geopoliméricos", Rev. Matéria 7, 3 (2002) 12.

[2] V. F. F. Barbosa, K. J. D. Mackenzie, C. Thaumaturgo, Int. J. Inorg. Mater. 2 (2000) 309-317.

[3] J. Davidovits, "30 years of successes and failures in geopolymer applications. Market trends and potential breakthroughs", in Geopolymer Conf., Melbourne, Austrália (2002).

[4] J. Davidovits, "Geopolymers: inorganic polymeric new materials”, J. Thermal Analysis 37 (1991) 1633-1656.

[5] J. Davidovits, "Geopolymers: Man-Made Rock Geosynthesis and the Resulting Development of Very Early High Strength Cement", in NASTS award 16 (1994) 91-139. [6] D. S. T. Pereira, F. A. Oliveira, F. J. Silva, C. Thaumaturgo, "Análise microestrutural de concreto geopolimérico: uma visão comparativa", Inter-Am. Conf. Non-Conventional Mater. Technol. Ecolog. Sustain. Constr. IAC-NOCMAT, Rio de Janeiro, RJ (2005).

[7] P. Duxson, J. L. Provis, G. C. Lukey, “Understanding the relationship between geopolymer composition, microstructure and mechanical properties", Colloid Surf. Phys. Chem. Eng. Aspects 269 (2005) 47-58.

[8] M. Steveson, K. Sagoe-Crentsil, "Relationships between composition, structure and strength of inorganic polymers. Part 1 - Metakaolin-derived inorganic polymers", J. Mater, Sci. 40 (2005) 2023-2036.

[9] ABNT - Associação Brasileira de Normas Técnicas, NBR NM 23, "Cimento Portland e outros materiais em pó - Determinação da massa específica”, Rio de Janeiro, RJ (2001).

[10] American Petroleum Institute, API SPEC 10A, "Specifications for Cements and Materials for Well Cementing" (2000).

[11] American Petroleum Institute, API SPEC 10B, "Recommended Practice for Testing Well Cements" (2000). [12] J. G. S.Van Jaarsveld, J. S. J. Van Deventer, G. C. Lukey, "The effect of composition and temperature on the properties of fly-ash and kaolinite-based geopolymers", Chem. Eng. J. 89 (2002) 63-73.

(Rec. 05/04/2015, Ac/ 15/05/2015) 